Federico Gobbo

Universiteit van Amsterdam

Davide Astori

Università degli Studi di Parma ${ }^{1}$

\title{
TRADUZIONE E DIACRONIA IN ESPERANTO: DANTE E PINOCCHIO
}

\section{Translation and diachrony in Esperanto: Dante and Pinocchio}

\begin{abstract}
Esperanto is the most successful planned language in history in terms of sociolinguistic relevance, as a community of practice formed around it since 1887. Esperanto proved its vitality by its diachronic change, limited but still present. Such change can be tested by comparing translations of the Divine Comedy and Pinocchio, published at different historical moments. This comparison illustrates the peculiarities of translating into Esperanto, a language that does not have an ethnic group of reference.
\end{abstract}

Keywords: Dante, diachrony, Divine Comedy, Esperanto, Pinocchio

\section{Riassunto}

L'esperanto è la lingua pianificata che ha avuto più successo nella storia in termini di rilevanza sociolinguistica, poiché si è formata una comunità di pratica a partire dal 1887. La vitalità dell'esperanto è provata dal suo cambiamento diacronico, limitato ma comunque presente. Tale cambiamento è verificabile mediante il confronto delle traduzioni della Divina Commedia e di Pinocchio, effettuate in momenti storici diversi. Questo confronto mette in evidenza le peculiarità traduttive e traduttologiche del tradurre in l'esperanto, lingua che non ha un gruppo etnico di riferimento.

Parole chiave: Dante, diacronia, Divina Commedia, esperanto, Pinocchio

1 Ai fini concorsuali, si noti che le sezioni 1, 2, 5 sono maggiormente dovute a Federico Gobbo, mentre le sezioni 3 e 4 sono maggiormente dovute a Davide Astori. La sezione 6 che chiude il contributo è dovuta in parti eguali ai due autori. 


\section{Introduzione}

Il tema della traduzione da e verso «lingue artificiali e morte» raramente viene affrontato con specificità nel dibattito scientifico ed accademico relativo alle scienze del linguaggio. In questo contributo intendiamo affrontare il tema della diacronia nella traduzione verso l'esperanto, la più nota delle lingue artificiali - per meglio dire, Plansprachen o lingue pianificate, seguendo la terminologia usuale in interlinguistica. A differenza delle lingue storico-naturali l'esperanto non è una lingua di origine etnica, e mostra quindi peculiarità di carattere traduttivo e traduttologico, che illustreremo qui.

L'accostamento tra lingue pianificate e lingue morte, per quanto non irragionevole, può essere foriero di fraintendimenti. Marcello Durante (1981: 1) descrive il passaggio dal latino all' italiano moderno, e delinea nel latino due volti: l'uno, quello a noi più familiare, è la fissità, vale a dire "un sistema di regole immutabili, "perpetuo e non corruttibile", riprendendo la definizione di Dante nel Convivio; l'altro, quello meno noto, è la mutevolezza, che "già si manifesta nel periodo protostorico [ma che] assume un ritmo assai più blando e sembra arrestarsi nell'età di Cicerone e di Cesare". A partire dal primo secolo avanti Cristo inizia quella che il Durante chiama 'la diaspora tra latino scritto e parlato' (p. V). Semplificando molto, sarà proprio il latino parlato dal volgo quello che darà la linfa vitale alle lingue romanze, tra cui l'italiano, mentre il latino scritto, fisso, normato, inizierà un lento ma inesorabile declino, e a poco a poco perderà vitalità: il volto della mutevolezza verrà preso dalle lingue romanze, mentre al latino rimarrà quel carattere di fissità che ci è più familiare. Va notato che esistono due gruppi di lingue umane che invertono l'ordine tra mutevolezza e fissità, partendo da quest'ultima: le lingue pianificate, come l'esperanto, e le lingue dei segni (sulle quali non ci soffermiamo in questa sede). In altri termini, a differenza delle lingue storico-naturali, le lingue pianificate acquisiscono prima il «volto della fissità» e solo in seguito, qualora vengano usate, cominceranno a mostrare il «volto della mutevolezza». Va menzionata inoltre l'altra anomalia specifica delle lingue pianificate: nella loro genesi, esse sono lingue non-etniche, perché pubblicate, nella fase iniziale di invenzione, da un singolo individuo, detto "glottoteta", o molto più raramente, da un comitato, ma mai da una comunità di parlanti (Gobbo, 2017).

\section{Diacronia e traduzione in esperanto}

Risulta dunque corretto affermare che le lingue pianificate siano il prodotto di una singola persona? Nel caso studio qui in esame, possiamo dire che l'esperanto è la lingua di Zamenhof? A rigore, tali affermazioni non sono del tutto corrette. Detlev Blanke $(2006 ; 1985)$ ha sviluppato una scala che mostra le tappe 
fondamentali dello sviluppo di una lingua pianificata, tappe che solo l'esperanto ha percorso completamente. In massima sintesi, il punto zero avviene quando il glottoteta esce dal segreto del suo studiolo e pubblica per iscritto il suo progetto di lingua pianificata, definendone il volto della fissità: ieri, mediante un libro stampato; oggi, mediante un sito web - la sostanza non cambia. A partire dal punto zero - che nelle lingue storico-naturali, lo ricordiamo, è una convenzione, mentre in quelle pianificate è chiaramente identificabile - la fase di invenzione linguistica si conclude e il glottoteta perde il controllo della lingua, che vedrà la sua evoluzione sottostando alle leggi tipici delle lingue storico-naturali.

Sempre secondo la scala di Blanke, i primi gradini consistono nell'usare la lingua prevalentemente per iscritto, per esempio nella corrispondenza o nei materiali didattici di base. Salendo su per la scala, si formerà una rete di sostenitori della lingua, riuniti prima in circoli cittadini, poi in associazioni, per arrivare infine a riconoscimenti da parte di istituzioni esterne anche internazionali, quali il PEN Club International, l'Unesco. Un gradino fondamentale è la formazione di famiglie che usano la lingua pianificata quotidianamente. Solo l'esperanto ha raggiunto questo stadio, con parlanti bilingue (e non monolingue) che la apprendono nel corso della prima socializzazione. Se la grande sfida delle lingue storico-naturali è la scrittura, la grande sfida delle lingue pianificate è l'oralità, e in ultima analisi solo l'esperanto può fregiarsi di averla vinta. Nella sua storia di poco più di centotrent'anni, l'esperanto ha prodotto un considerevole corpo di opere letterarie (soprattutto del primo tipo, vale a dire belletristica), sia tradotte che originali. Esiste un'opera poderosa a carattere enciclopedico, pubblicata in inglese, che descrive la letteratura originale in esperanto (Sutton, 2008). Lo studioso notava che

la cultura esperanto probabilmente è tra le più aperte ed accessibili culture del mondo poiché i creatori sono rappresentativi di vari popoli e nazioni, e nonostante la loro inevitabile conformità alle tradizioni della giovane cultura esperanto, apportano in essa motivi e costituenti delle culture nazionali a loro proprie (Gobbo, 2009: 118).

Un'altra opera sulla storia della letteratura esperanto, scritta in lingua, propone una lettura critica di opere originali e in traduzione (Minnaja, Silfer, 2015). Emerge in entrambe le letture che nella sua fase pionieristica (1887-1921) è la letteratura, specie in traduzione, a guidare i parlanti a trovare la confidenza necessaria per parlarla.

Visto lo statuto così importante della traduzione nella formazione dello stile, vogliamo mostrare la vivezza dell'esperanto attraverso l'analisi comparata e contrastiva di diverse traduzioni, effettuate in momenti differenti nella storia di questa lingua, di due opere classiche della letteratura italiana: la Divina Commedia di Dante e Pinocchio di Carlo Collodi. Avere più traduzioni, a distanza 
di tempo, della stessa opera, è un modo per toccare con mano l'evoluzione di una lingua, soprattutto nella resa del parlato - per esempio nei dialoghi - che è portatore delle innovazioni linguistiche, vale a dire mostra il volto della mutevolezza.

\section{Dante e la Divina Commedia in esperanto}

Bruno Migliorini (1973: 449) si domanda, retoricamente, perché tradurre in esperanto la Divina Commedia, e la risposta risiede nella universalità del messaggio dantesco. La resa in esperanto della Divina Commedia è un'epopea straordinaria. Il panorama delle traduzioni in esperanto della Commedia vanta una vasta messe di traduzioni di parti del poema; per motivi di spazio, ci si concentrerà sulle traduzioni più rilevanti: quella dell'Inferno di Kálmán Kalocsay, e le due traduzioni integrali.

Kálmán Kalocsay, di famiglia nobile ungherese, specialista in malattie infettive in un grande ospedale di Budapest, docente universitario, redattore capo della rivista Literatura mondo, poliglotta, mostra da subito la sua maestria di traduttore con numerose traduzioni di poesia e prosa. Seguendo le orme del suo maestro Mihály Babits, traduttore della Divina Commedia in ungherese, Kalocsay pubblica prime versioni in esperanto di parti della cantica, a partire dal V canto, fino a che non vedrà la luce la traduzione integrale, pubblicata su Literatura mondo nel 1933.

Dovranno passare altri trent'anni prima che la prima traduzione integrale del capolavoro dantesco in esperanto possa vedere la luce. Dal Regno Austroungarico a oggi, Trento è caratterizzata dalla convivenza delle etnie e delle rispettive lingue su quel territorio. In tale clima spicca la figura poliedrica di Giovanni Peterlongo, sindaco della città e successivamente Commissario prefettizio (Astori, 2015a). Accanto al suo impegno politico vive parallelamente una personale esperienza di esperantista, e traduce in lingvo internacia la Divina commedia. Serenella Baggio (2015: 16-17) nota che per Trento Dante fu non solo "icona risorgimentale" ma anche "dell'irredentismo trentino in particolare", e che fu proprio Peterlongo a inaugurare il monumento cittadino a Dante nel 1896. La traduzione, già probabilmente completata in prima stesura nel 1914, vede la luce solo nel 1963, grazie al figlio Paolo. L'edizione sarà bilingue, e uscirà a seguito di una lunga revisione, cui parteciparono i fratelli Migliorini, già revisori dell'Infero di Kalocsay, ed Enrico Dondi, di cui si dirà subito oltre, ma anche altri, fra cui non ultima Clelia Conterno Guglielminetti, che dà un più approfondito del lavoro di revisione nel 1973.

Ci vorranno quasi altri cinquant'anni prima di avere la seconda traduzione integrale del capolavoro dantesco. Alla fine degli anni Cinquanta, Enrico Dondi, all'epoca studente di medicina, poi neurologo, comincia a cimentarsi 
con la traduzione di Dante. Dopo l'impresa di Kalocsay, inizialmente tralascia l'Inferno, e fornisce, del Purgatorio, alcune parti, che confluiranno nella Antologia italiana, del 1987. Sarà anche traduttore delle canzoni del Convivio e della Vita nova, la quale uscirà nel 2003. La versione completa in esperanto del Purgatorio è probabilmente realizzata alla fine degli anni Cinquanta, anche se vedrà la luce solo nel 2000, in grande formato, in un'edizione bilingue. Nel 2006, viene pubblica l'intera Commedia, in tre volumi, questa volta senza testo originale, con una nuova edizione anche del Purgatorio. Riproduciamo qui uno specimen dell'incipit dell'opera dantesca con le tre versioni in esperanto.

Nel mezzo del cammin di nostra vita mi ritrovai per una selva oscura, ché la diritta via era smarrita.

Ahi quanto a dir qual era è cosa dura esta selva selvaggia e aspra e forte che nel pensier rinova la paura!

Tant'è amara che poco è più morte; ma per trattar del ben ch'i' vi trovai, dirò de l'altre cose ch'i' v' ho scorte.

En mezo de l' vojaĝ' de nia vivo en arbareg' malluma mi troviĝis, ĉar mi de l' rekta vojo forvojiĝ̀is.

Ha kiom pezas diri kia estis Tiu arbar' sovaĝa, kruda, densa, kiu eĉ pense renovigas timon!

Amaras gì, kaj mort' malmulte plias; sed, por pritrakti la trovitan bonon, mi diros l’aliajojn ekviditajn.

(Peterlongo, 1963)
Je l' vojomez' de nia vivo tera mi trovis min en arbareg' obskura, car perdiginta estis vojo vera.

Ho, ve eĉ diri, kia lok' terura estas ĉi kruda, pena dens' arbara, revekas timon eĉ memoro nura.

Eĉ mort' apenaŭ estas pli amara. Sed traktos mi pri renkontita ciio, por ke l' trovita Bono iĝ̀u klara.

(Kalocsay, 1933)

En mezo de la voj' de vivo nia mi trovis min en arbareg' obskura, de l' rekta voj' estinte fordevia.

Ho ve, raporti estas takso dura, kiom l' arbaro krudas kaj malbonas; $\hat{g} i$ eĉ en penso estas plej terura.

Pli da amaro, nur la morto donas, sed pro la bono, kiun mi entrovis, pri l' ajoj travivitaj mi eksponas.

(Dondi, 2006)

\section{Tre stili, tre Danti}

Difficile sintetizzare una valutazione delle tre proposte traduttive presentate in momenti diversi della storia novecentesca: ci limitiamo ad alcuni cenni. Kálmán Kalocsay, "la stella più brillante del firmamento esperantofono”, 
secondo Carlo Minnaja (2015: 69), fra i più competenti critici letterari esperanto, è "traduttore molto fedele, ma poeta egli stesso". Kalocsay "accoglie nuovi vocaboli con naturalezza" e allenta la metrica un po' rigida dell'iniziatore dell'esperanto, Zamenhof, avvicinandola "quasi a sovrapporsi a quella italiana". Venendo alla seconda versione, Minnaja fa notare che Giovanni Peterlongo non ebbe contatti con altri esperantofoni, e quindi "rimase quindi un isolato, anche dal punto di vista linguistico". Il suo intento era rendere la Divina Commedia "accessibile a tutti i ceti". Per questo motivo, contrariamente a Kalocsay, preferisce "utilizzare gli affissi su radici di base piuttosto che quei neologismi che man mano le esigenze letterarie andavano proponendo nell'esperanto" (Corsini, 1969). Minnaja (2015) nota che, a differenza di Kalocsay, era ancora la traduzione dell'Amleto pubblicata da Zamenhof nel 1894 ad essere il punto di riferimento del Peterlongo, e di qui la metrica scelta, di matrice anglo-germanica, anziché italiana.

Relativamente a Enrico Dondi, Nicolino Rossi (2012), raffinato traduttore e poeta in esperanto, nel suo necrologio, scritto in esperanto, ebbe ad affermare (traduzione degli autori):

questa dolce fluidità dell'endecasillabo classico, dal ritmo morbido, che Dondi restituisce con un ritmo regolare ma non monotono, preferendo il piede giambico, ci fornisce versi dal respiro largo, leggeri e sonori, che sottolineano virtuosamente la densità semantica intesa da Dante.

Mentre Kalocsay dovette introdurre molti vocaboli nuovi, il che gli procurò l'ostilità di chi propagandava la facilità di apprendimento dell'esperanto nella limitatezza del suo lessico, Dondi si trova in un contesto con un registro alto, letterario e scientifico, dell'esperanto, già molto più evoluto. Dondi è più traduttore che poeta, a differenza dell'ungherese: "le rime appaiono più semplici, quasi naturali: potremmo dire che il suo limite è nel fatto che egli rende Dante un poeta quasi facile, alla portata di tutti” (Minnaja, 2015).

\section{Pinocchio in esperanto: due traduzioni, due scopi programmatici}

Esistono due traduzioni in esperanto del capolavoro collodiano: la prima è a cura di Mirza Marchesi (1930) mentre la seconda, uscita a distanza di oltre settant'anni dalla prima, è a cura di Jozefo Horváth (2003), pseudonimo di Giuseppe Croatini (Astori, 2015b). Come nel caso di Dante, la scelta di rendere in esperanto Pinocchio è tutt'altro che casuale: l'idea di mondoliteraturo, letteratura mondiale, nella cultura esperanto assume un significato diverso dal significato di Weltliteratur. Se Goethe auspicava la circolazione in traduzione delle opere massime delle letterature nazionali perché il loro messaggio trascende 
i confini nazionali per abbracciare l'umanità, l'idea sottesa all'impresa iniziata da Zamenhof è quella di mettere in comune i tesori nazionali per formare una nuova cultura mondiale su base non-nazionale mediante una lingua comune. Come visto in precedenza, Bruno Migliorini aveva sottolineato l'universalità di Dante giustificandone così la resa in esperanto; analogamente per il Pinocchio della Marchesi. Nel caso di Joseph Horváth, la traduzione ha lo scopo di dare al pubblico esperantofono una traduzione più moderna (Minnaja, 2003). La recensione di Nordenstorm (2012) a questa seconda traduzione mette in rilievo l'uso di espressioni in lingua, di sapore proverbiale, quale mankas klapo en la kapo per rendere 'qualche grillo per il capo'. Questo testimonia chiaramente che il volto della mutevolezza è ben sviluppato, all'alba del nuovo millennio, nell'esperanto, che dispone non solo di un solido e ampio dizionario monolingue dal 1970, di fatto il riferimento obbligato per tutti i parlanti, ma anche di una tradizione di stile che ha permesso l'emergere di figure stilistiche e di metafore lessicalizzate, passando attraverso momenti di fluidità, tra versioni parallele in competizione (Astori, 2017). Se lo scopo del 1930 è arricchire la letteratura mondiale in un'ottica tradizionalmente esperantista, quello del 2003 intende invece mostrare, attraverso il frequente uso di formazioni endogenetiche, come la lingua si sia arricchita moltissimo a partire dal secondo dopoguerra, al punto tale che la traduzione di Horváth per molti versi non sembra tale, come ogni buona traduzione dovrebbe essere. Nel tirare le somme sul confronto e contrasto tra le due traduzioni in esperanto del capolavoro collodiano, Astori (2016) rileva tuttavia che esiste una questione aperta nell'esperanto contemporaneo, rappresentata dalla ancora scarsa riconoscibilità di diversi registri linguistici, attributo indispensabile per l'adeguata resa traduttiva. Tale osservazione può essere estesa per una riflessione più generale.

\section{Riflessioni conclusive}

La questione dei registri linguistici in esperanto è ancora aperta. Non intendiamo qui la presenza o meno di lessici specialistici, per esempio per gli ambiti tecnici o la comunicazione scientifica. L'esperanto, in molte discipline, possiede dizionari specialistici o letteratura di settore. Intendiamo invece la resa diversa dei registri del parlato, per esempio dimesso, aulico, colloquiale, o anche strafottente. Le tre interpretazioni traduttive del capolavoro dantesco mostrano come sia possibile rendere in esperanto un registro poetico originale, ricco di invenzioni (Kalocsay) oppure, al contrario, usare per tradurre un registro più piano, di immediata comprensibilità (Peterlongo e Dondi). L'italiano possiede una varietà di registri straordinaria, mentre la varietà di registri dell'esperanto è assai minore. Limitandoci a un cenno alla dimensione diatopica, l'italiano ottiene tale grandiosa varietà principalmente grazie al contatto con le lingue 
regionali e minoritarie di tradizione. In esperanto, invece, la dimensione diatopica non vuole essere positivamente rilevante, perché le interferenze con le lingue nazionali vengono viste, a torto o a ragione, come mere varietà d'apprendimento, di passaggio, da superare. Lo scopo è raggiungere il registro medio non marcato, che l'esperanto, nel giro di cinque generazioni (125 anni) è riuscito, finalmente, a definire, tra molte difficoltà e tentennamenti. Riprendendo la metafora del Duranti, il volto della mutevolezza nell'esperanto c'è, ed è rappresentato dalla variabilità dei registri nell'uso parlato. Tuttavia, esiste una incertezza diffusa da parte degli esperantofoni sul volto della mutevolezza, e quindi tale volto rimane per lo più nascosto. Sono purtroppo ancora pochi i casi di più traduzioni effettuate a distanza di tempo delle stesse opere, quali quelli esaminati. L'analisi traduttiva e traduttologica delle traduzioni a confronto mostra infatti la realtà dello sviluppo diacronico di una lingua. Essa diventa una prova tangibile dello statuto di lingua viva.

Proprio questa incertezza fa sì che gli esperantisti provino a mostrare il volto della mutevolezza della loro lingua attraverso espedienti letterari per creare registri diversi. Per esempio, Manuel Halvelik ha inventato una lingua classica fittizia, l'arcaicam esperantom, da cui sarebbe derivato l'esperanto, analogamente al ruolo del latino per l'italiano e le altre lingue romanze (Astori, 2013). Ma si tratta, per l'appunto, di espedienti dotti, colti, artificiali.

Ci sembra più interessante trovare la vitalità dell'esperanto attraverso le diverse traduzioni in esperanto dei grandi classici della letteratura qui presentati, effettuate in momenti diversi della storia della lingua iniziata da Zamenhof e realizzata dalla collettività esperantofona. Concludiamo l'intervento dichiarando che l'esperanto non può essere assolutamente considerato una lingua morta ma al contrario si tratta di una lingua viva e vitale.

\section{Bibliografia}

ANON. Kalocsay, Kalman. In: Enciclopedia Dantesca, vol. III, p. 538. Roma: Istituto della Enciclopedia Italiana Treccanip.

Astori, D. (2013): How “to age” a language: the 'arcaicam esperantom' of Manuel Halvelik/ Come "invecchiare” una lingua: l'arcaicam esperantom' di Manuel Halvelik/ Kiel “maljunigi” lingvon: la 'arcaicam esperantom' de Manuel Halvelik. In: Barandovska-Frank, V. (a cura di), Litera scripta manet. Serta in honorem Helmar Frank, pp. 97-111. Paderborn-Praha: Akademia Libroservo.

Astori, D. (a cura di) (2015a): Giovanni Peterlongo. L'uomo, il politico, l'esperantista. Trento: Regione Autonoma Trentino-Alto Adige.

Astori, D. (2015b): "Pinokjo": tradurre Collodi in una lingua pianificata. In: Busà, M.G., Gesuato, S. (a cura di), Lingue e contesti. Studi in onore di Alberto M. Mioni, pp. 951-961. Padova: Cleup. 
Astori, D. (2016): Le traduzioni di "Pinocchio": come si è evoluto l'esperanto tra il 1930 e il 2003. In: Gobbo, F. (acd / red / ed), Lingua, politica, cultura: Serta gratulatoria in honorem Renato Corsetti, pp. 150-161. New York: Mondial.

Astori, D. (2017): Metafore nell'esperanto: una cartina al tornasole eterodossa della moderna riflessione più tradizionale sulle lingue naturali. In: Astori, D. (a cura di), La metafora e la sua traduzione fra riflessioni teoriche e casi applicativi, pp. 133-148. Parma: Bottega del Libro editore.

Baggio, S. (2015): La situazione linguistica del Trentino. In: Astori, D. (a cura di), Giovanni Peterlongo. L'uomo, il politico, l'esperantista, pp. 15-51. Trento: Regione Autonoma Trentino-Alto Adige.

Blanke, D. (1985): Internationale Plansprachen: Eine Einführung. Berlin: Akademie Verlag.

Blanke, D. (2006): Interlinguistische Beiträge. Zum Wesen und zur Funktion internationaler Plansprachen, Hrsg. von S. Fiedler. Frankfurt am Main: Peter Lang.

Conterno Guglielminetti, C. (1973): Kiel ni reviziis la ĉefverkon. Literatura foiro, 4 (20), pp. 4-8.

Corsini, U. (1969): Giovanni Peterlongo e una versione in esperanto del poema dantesco. Studi trentini di scienze storiche, XLVIII (2), pp. 113-118.

Dondi, E. (2006): Dante Alighieri, La Dia Komedio. Tre volumi. Illustrazioni di Gustavo Doré. Chapecó (Brasile): Fonto.

Durante, M. (1981): Dal latino all'italiano moderno. Bari: Laterza.

Gobbo, F. (2009): Fondamenti di interlinguistica ed esperantologia: pianificazione linguistica e lingue pianificate. Milano: Raffaello Cortina.

Gobbo, F. (2017): Are Planned Languages Less Complex than Natural Languages? Language Sciences, 60, pp. 36-52.

Horváth, J. (2003): Traduko de C. Collodi, La aventuroj de Pinokjo. Antwerpen: Flandra Esperanto-Ligo.

Kalocsay, K. (1933): D. Alighieri, Infero. Budapest: Eldono de Literatura Mondo.

Marchesi, M. (1930): Traduko de C. Collodi, La aventuroj de Pinokjo. London: the Esperanto Publishing Company Ltd.

Migliorini, B. (1973): Peterlongo, Giovanni. In: Enciclopedia Dantesca, vol. IV, pp. 449450. Roma: Istituto della Enciclopedia Italiana Treccani.

Minnaja, C. (2003): Marioneto ciiam aktuala (recensione a Horváth, J. (2003): Traduko de C. Collodi, La aventuroj de Pinokjo. Antwerpen: Flandra Esperanto-Ligo), Monato, 6, p. 20.

Minnaja, C. (2015): Le traduzioni di Dante in esperanto. In: Astori, D. (a cura di), Giovanni Peterlongo. L'uomo, il politico, l'esperantista, pp. 69-80. Trento: Regione Autonoma Trentino-Alto Adige.

Minnaja, C., Silfer, G. (2015): Historio de la Esperanta Literaturo. La Chaux-de-Fonds: LF-Koop.

Nordenstrom, L. (2012): Infanlibro en ne tro komplika lingvajo (recensione di Horváth, J. (2003): Traduko de C. Collodi, La aventuroj de Pinokjo. Antwerpen: Flandra Esperanto-Ligo), http://katalogo.uea.org/katalogo. php?inf=6947\&id=1434\&recenzo=montru\#ek (accesso: 7.04 .2014$)$. 
Peterlongo, G. (1963): Dante Alighieri, La Divina Commedia / La Dia Komedio, versione con testo a fronte e commento in esperanto di Giovanni Peterlongo con illustrazioni di Sandro Botticelli. Serie Oriente-Occidente - Edizione speciale sotto gli auspici della Federazione Esperantista Italiana, dell'Istituto Italiano d'Esperanto e dell'Associazione Universale Esperantista, in relazioni consultive con la U.N.E.S.C.O. Milano: Edizione SIEI (deposito e vendita in Firenze presso la Casa Editrice Le Monnier).

Rossi, N. (2012): Funebre pri D-ro Enrico Dondi, 12 gennaio 2012, https://groups. google.com/forum/\#!topic/labor-gruporevuo/4-CisWU8s58 (accesso: 15.01.2012).

Rowling, J.K. (2003): Harrius Potter et PHILOSOPHI Lapis. London: Bloomsbury.

Stria, I. (2015): Towards a Linguistic Worldview for Artificial Languages. Rozprawa doktorska napisana pod kierunkiem prof. UAM dr hab. Ilony Koutny. Poznań: Uniwersytet im. Adama Mickiewicza.

Sutton, G.H. (2008): Concise Encyclopedia of the Original Literature of Esperanto. New York: Mondial.

Wüster, E. (1978): Esperantologiaj studoj, a cura di R. Haupenthal. Stafeto: La Laguna. 